\title{
USING OF NEW CONTROL METHODS OF EFFICIENCY OF HIPPOTHERAPY FOR CHILDREN WITH SINGULARITIES OF PSYCHOPHYSICAL DEVELOPMENT
}

\author{
Tatiana Tereshrova, Ludmila Loseva \\ Yanka Kupala State University of Grodno, Grodno, Belarus
}

\begin{abstract}
The number of disabled people is increasing, and the problem of their rehabilitation and social adaptation is important. In the Republic of Belarus, hippotherapy began to be applied about ten years ago. Within this time, about 200 children sick with cerebral spastic infantile paralysis (severity levels I-III), epilepsy, autism and other diseases underwent rehabilitation. About 70 of them started moving independently; the disability degree of 25 children was lowered or removed.

The study on using the method of X-ray fluorescent analysis (RFA) during hippotherapy was the first of its kind in the Republic of Belarus.

The aim of the research was the detection of efficiency of impact of hippotherapy on children with psychophysical peculiarities with use of modern control methods.

The need for correction of the following elements - calcium, potassium, ferrum, cuprum, zinc, selenium, iodine - in children's ration at the time of hippotherapy sessions was shown. The need for increasing the frequency of sessions of hippotherapy from once a week to three times a week was established.
\end{abstract}

Keywords: hippotherapy; children; X-ray fluorescent analysis

\section{INTRODUCTION}

Both world and domestic statistics confirm the scale of the problem of children's invalidisation and the need for its quick solution. Nowadays, a sustainable tendency of increase in the number of disabled children with cerebral paralysis 
is observed. While in 1962, according WHO data, 0.4 cases per 1000 children were registered, in 1982, this figure amounted to 5.6 and, in 1992, to 9 cases per 1000 children.

Since the beginning of 2000 to the present, $13-15$ cases of cerebral infantile paralysis have been registered per 1000 newborns, while spastic forms predominate in its occurrence frequency. The number of disabled people is increasing, and the problem of their rehabilitation and social adaptation is important. Even in ancient times people noticed the medical properties of riding and the favourable impact of communication of patients with horses. In 1791, the philosopher Denis Diderot wrote that "among the physical exercises possessing all fine qualities, the first place belongs to riding, which treats a number of diseases..."

In the Republic of Belarus, hippotherapy began to be applied about ten years ago. At this time, the Belarusian Public Association of Riding and Hippotherapy (henceforth BPAR\&H) was established. Here, more than 30 children are being rehabilitated annually. This is a large number of patients for such a small centre. BPAR\&H was the first society in Belarus in which hippotherapy started to be practiced. Our as well as western experts had to overcome the scepticism of doctors and mistrust of horse-riding professionals. BPAR\&H has existed since 1998 and has been a member of medical riding and equestrian sport for the disabled of the Russian Federation since 2000. During this time, about 200 children with cerebral spastic infantile paralysis (severity levels IIII), epilepsy, autism and other diseases have undergone rehabilitation. About 70 of them started moving independently, the disability degree of 25 children was lowered or removed.

The study on using the method of X-ray fluorescent analysis (henceforth RFA) during hippotherapy was the first of its kind in the Republic of Belarus.

The aim of the research was the detection of efficiency of the impact of hippotherapy on children with psychophysical peculiarities with the use of modern control methods.

\section{MATERIALS AND METHODS OF RESEARCH}

It is known that it is necessary to support the daily balance of vitamins, macroand microelements in the human body, especially of children [1-5]. Multielement analysis of children's hair during hippotherapy was conducted by means of the X-ray fluorescent method. Hair was cut from 3-5 places on the occipital part of the head.

Twenty children and teenagers of different gender and age (3-17 years) of the "House for the disabled children" of the town of Rechitsa took part in 
bench supervision. The base for classes of hippotherapy was the horse-racing complex of Ozershchina of Children's and Youth Sports School No. 2 in the department of physical culture, sports and tourism, the Rechitsa administrative district of the Gomel region. Research of biomaterial (hair, wool) on the estimates of the endo-ecological status of the groups of supervision was carried out on the basis of the laboratory of physical and chemical methods of research of environment objects at Yanka Kupala State University of Grodno. Pedagogical supervision was implemented by the department of theory and methods of physical culture [6-7].

\section{Advantages of the method:}

the possibility of receiving the review range of all the elements with one measurement;

the speed of obtaining information;

minimum sample preparation without sample destruction;

studying of samples in various matrices;

small consumption of energy and reactants;

the opportunity of carrying out repeated measurements.

\section{Preparation of samples for measurement}

Preparation of samples for measurement

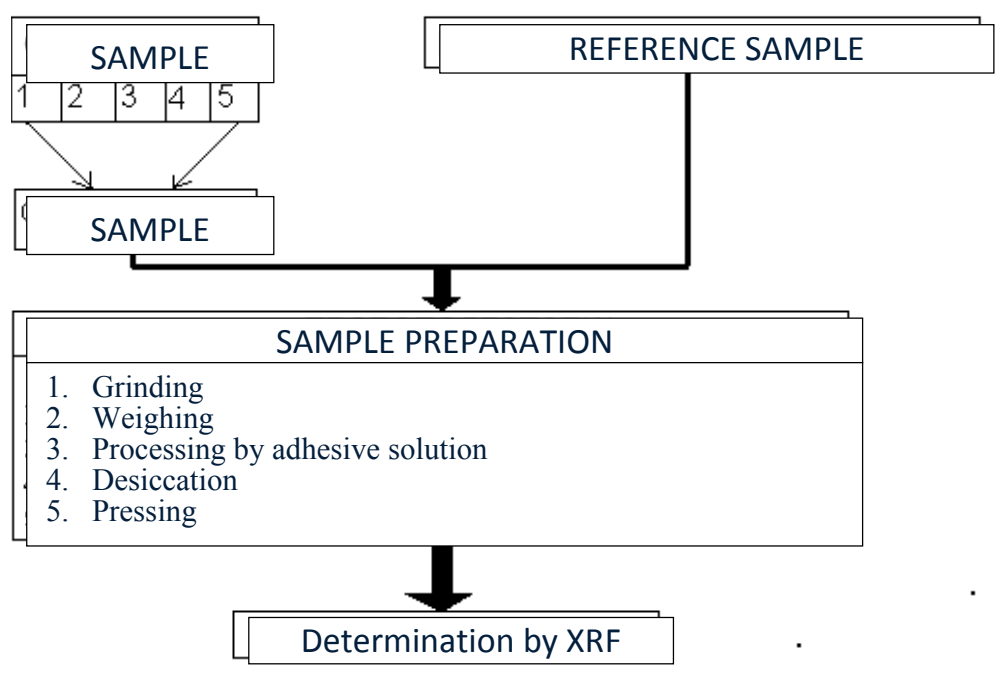

Figure 1. Stages of preparation of the biomaterial sample (hair).

During supervision, the obtained data were processed by the methods of mathematical statistics and are presented in the Table and in Figures 2-5 [8]. 


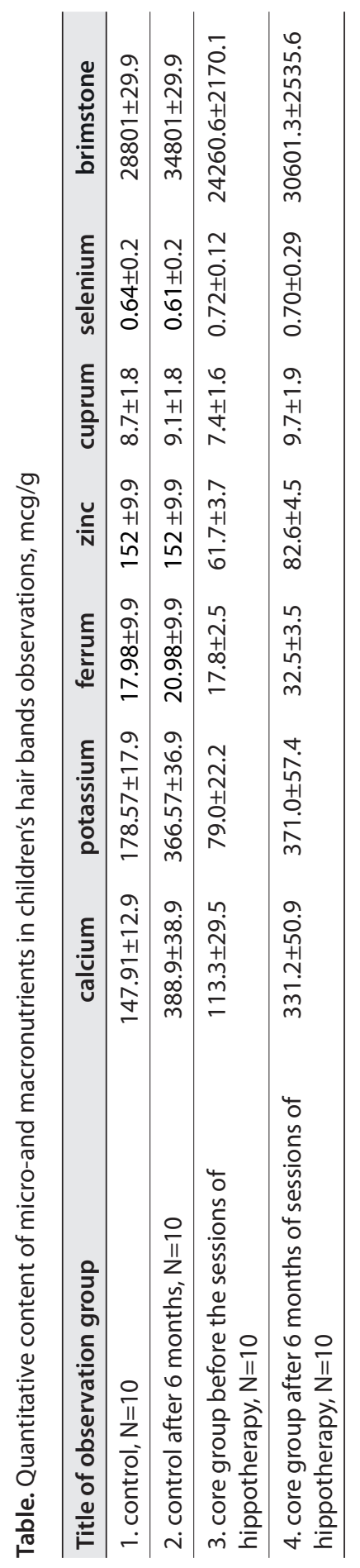




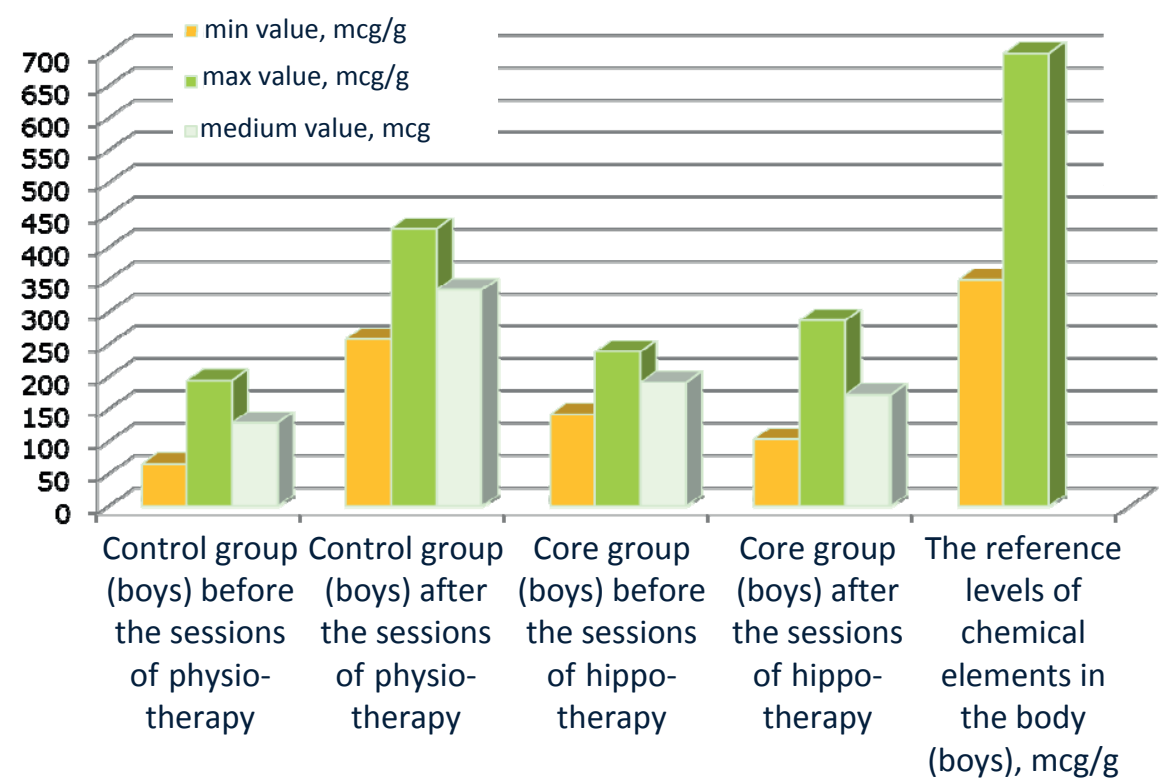

Figure 2. Quantitative content of calcium in boys' body (hair) before and after therapy, $\mathrm{mcg} / \mathrm{g}$.

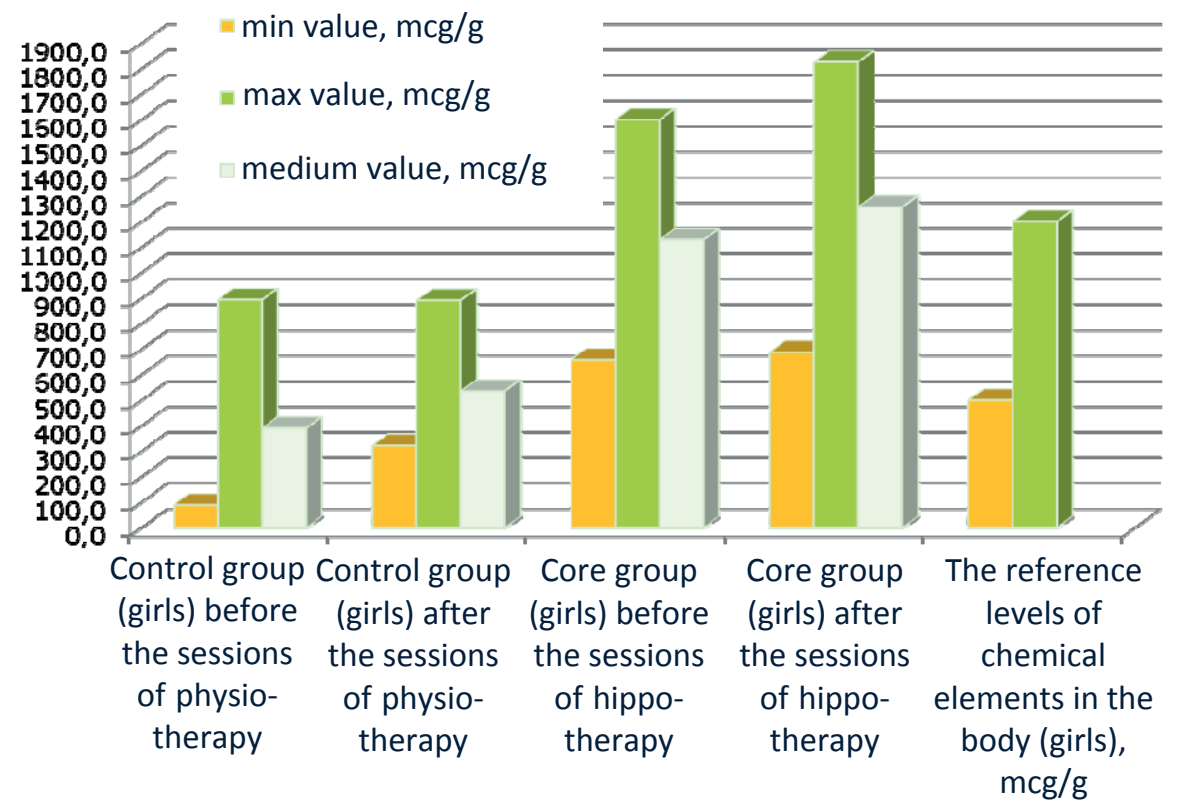

Figure 3. Quantitative content of calcium in girls'body (hair) before and after therapy, mcg/g. 


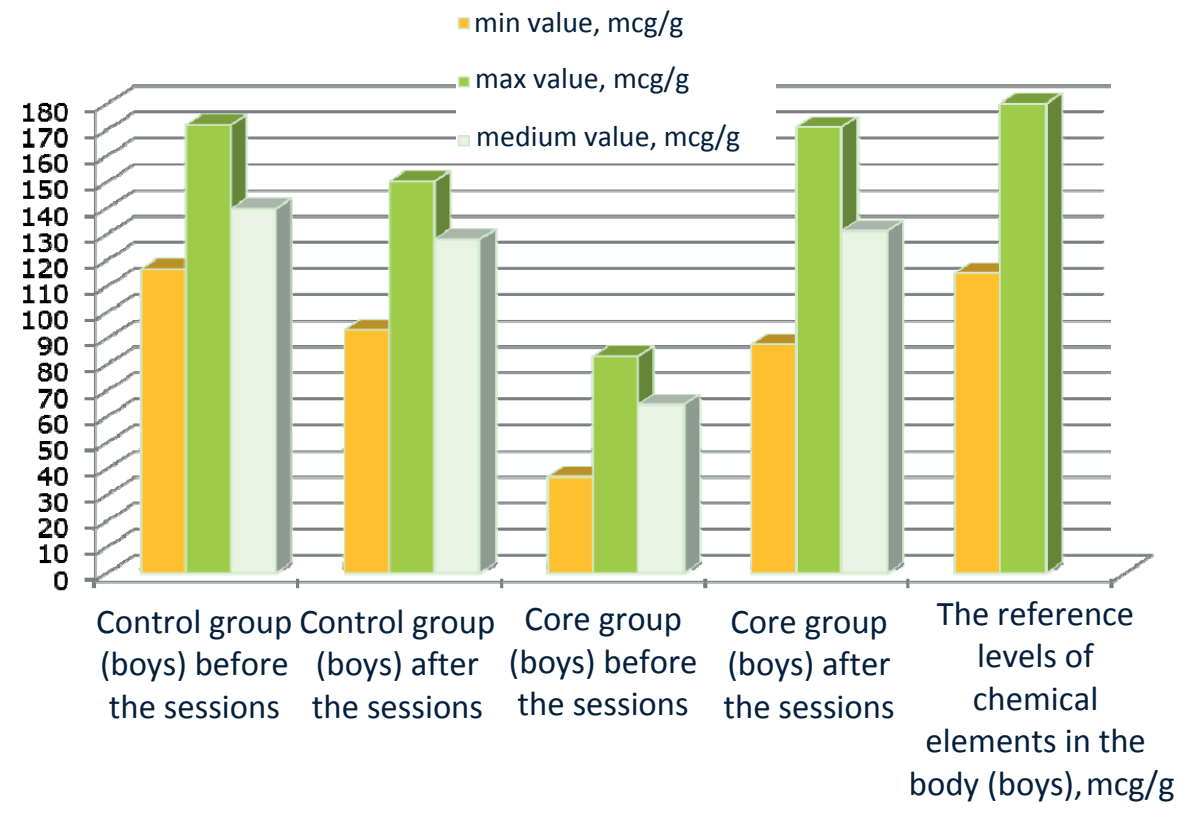

Figure 4. Quantitative content of zinc in boys' body (hair) before and after therapy, mcg/g.

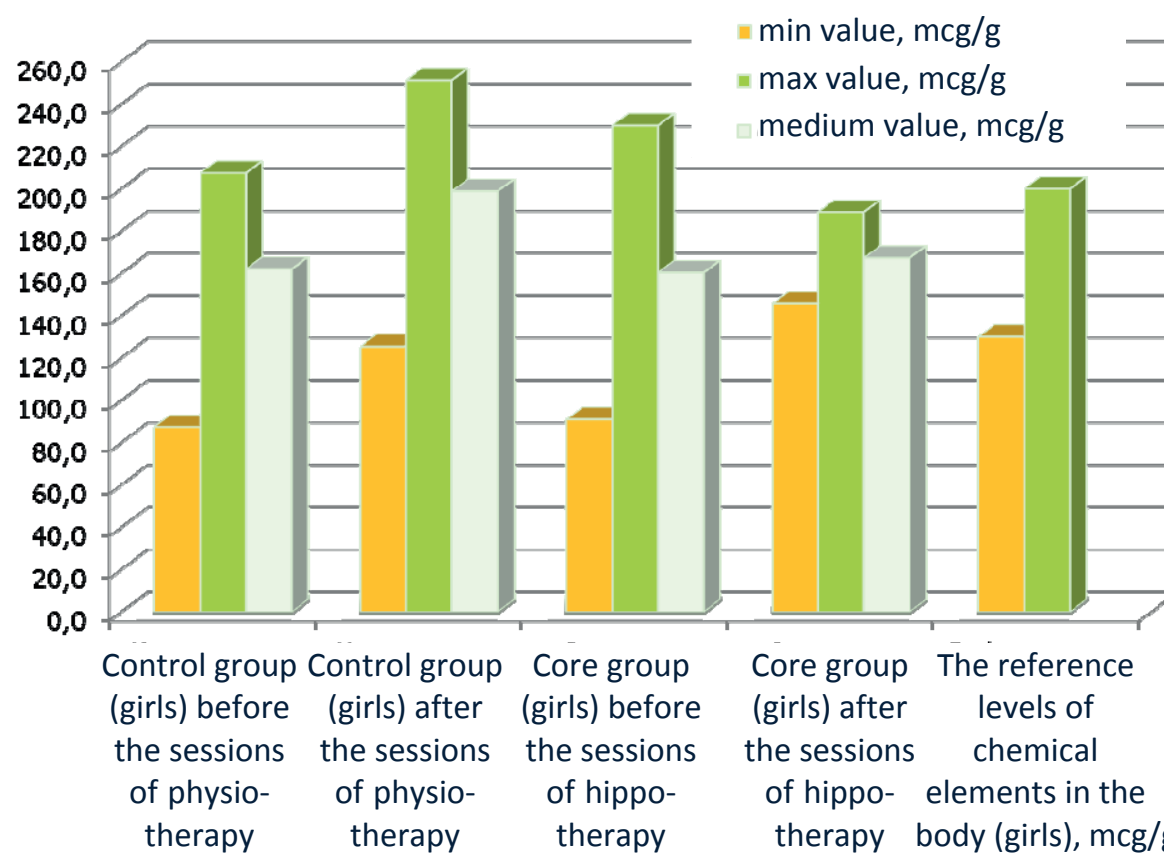

Figure 5. Quantitative content of zinc in girls body (hair) before and after therapy, mcg/g. 


\section{CONCLUSIONS}

1. For the first time the possibility of application of the methods of pedagogical and X-ray fluorescent analysis as the control of assessment of efficiency of hippotherapy in rehabilitation practice was shown.

2. In the course of the sessions of hippotherapy, positive dynamics of essential elements - calcium, zinc, selenium, and ferrum - was established.

3. The need of correction of children's ration at the time of sessions of hippotherapy of the following elements: calcium, potassium, ferrum, cuprum, zinc, selenium, iodine was shown.

4. The need for increasing the frequency of sessions of hippotherapy from one time a week to three times a week was established.

\section{REFERENCES}

1. Adaptive physical culture at work with the children with violations of the musculoskeletal device (at a disease of a children's cerebral palsy): methodical tutorial / editor A.A. Potapchuk. SPB: P.F. Lesgaft SPBGAPC.

2. Arkhipova E.F. (1989). Correctional work with children with cerebral palsy [An electronic resource] M.: Education. Access mode: www.pedlib.ru

3. Atmadzhan A. (1992). The intellectual pathology, the suffering individual and a horse, Paris.

4. Badalyan L.O. (1988). Children's cerebral paralysis. L.O. Badalyan, L.T. Zhurba, O.V. Timonina. Kiev: Health. 328 p.

5. Badalyan L.O. (1984). Pediatric neurology. L.O. Badalyan. M.: Medicine. 426 p.

6. Loseva L.P., Poznyak S.S., Fus S.V., Kirilenko E.K. (2008). New possibilities of research of a condition of microelement balance. Collection of works of the 8th international scientific conference "Sakharov readings 2008: environmental problems of the XXI century". Minsk, 25-28.

7. Pushkareva M.N., Losev L. P., Paramonova N. A. (2010). An element homeostasis and problems of children's and teenagers' adaptation in the conditions of increased physical activity. Collection of articles "Health and environment", 60-65, Minsk.

8. Skalny A.V. (2004). Chemical elements in physiology and ecology of the person. M., Onyx 21 century, World. p. 215

9. Samarsky V.V., Kuzmenko N.Y. Hippotherapy: Features of application, development problem. Wellness horse centre "Solar Island". St. Petersburg, 62-65.

10. Shamro E.V. Using hippotherapy at rehabilitation of children with perinatal defeat of the central nervous system. Regional Public Organization "Center of mutual integration "Chord"', St. Petersburg. 103 p. 
94 | T. Tereshkova, L. Loseva

Address for correspondence:

Ludmila Pavlovna Loseva

Pulichova 31/1, app.116

220088 Minsk

Belarus

E-mail: Spirulina1945@gmail.com 